American Medical Journal 1 (2): 157-163, 2010

ISSN 1949-0070

(C) 2010 Science Publications

\title{
Evaluation of Nested PCRs targeting the BI and SAG2 Genes for Detection of Toxoplasma gondii Genome in Aqueous Humor from HIV Positive Toxoplasma Retinochoroiditis Patients in a Tertiary Eye Hospital
}

\author{
${ }^{1}$ B. Mahalakshmi, ${ }^{1}$ K.L. Therese, ${ }^{1}$ R. Kirthika, ${ }^{1}$ H.N. Madhavan, ${ }^{2}$ J. Biswas and ${ }^{2}$ S. Sudharshan \\ ${ }^{1}$ Larsen and Toubro Microbiology Research Centre, Vision Research Foundation, \\ Chennai, Tamil Nadu, India \\ ${ }^{2}$ Department of Uvea, Sankara Nethralaya, Chennai, Tamil Nadu, India
}

\begin{abstract}
Problem statement: To evaluate nPCR targeting B1 gene (primer set 1) with 3 other nPCRs targeting B1 gene (primer set 2), 3' end of SAG2 and 5' end of SAG2 gene, for detection of Toxoplasma gondii (T. gondii) genome on AH from HIV positive TRC patients. Approach: DNA extracted from AH of 12 TRC patients and 12 controls (patients with ocular inflammation of nonToxoplasma origin) were subjected to all the 4 nPCRs. Results: Toxoplasma gondii genome was detected by atleast one of the $4 \mathrm{nPCRs}$ in $8(66 \%)$ of 12 TRC patients and in none of the 12 controls. nPCR targeting B1 gene (primer set 1) was positive in 6, B1 gene (primer set 2) in 2 and both 3 'end of SAG2 and 5' end of SAG2 in 4 respectively. The sensitivity of B1 gene (primer I) was higher compared to the other $3 \mathrm{nPCRs}$ (Yates correction-Chi square test; $\mathrm{p}=0.028$ ). Conclusion: Among the 4 nPCRs, nPCR targeting B1 gene (primer set 1) was the most sensitive and reliable nucleic acid amplification technique for the laboratory diagnosis of TRC in HIV patients.
\end{abstract}

Key words: Toxoplasma retinochoroiditis, B1 gene, HIV positive, polymerase chain reaction, SAG2 gene, aqueous humor

\section{INTRODUCTION}

Toxoplasma gondii (T. gondii), a protozoan parasite, can cause severe, life threatening disease, especially in newborns and immunosuppressed patients and is an important cause of ocular disease in both immunosuppressed and immunocompetent individuals (Holland, 1999). T. gondii may affect the brain, eye as well as the lung in case of individuals with AIDS/HIV, malignancy and organ transplant. Toxoplasma retinochoroiditis (TRC) is believed to account for 1-3\% of retinal infections in patients with AIDS (Jabs et al., 1989; Tabbara, 1990; Cochereau-Massin et al., 1992).

Early and appropriate treatment of ocular toxoplasmosis in AIDS patient is vital, because not only is the ocular prognosis good, but also because ocular disease often coexists with life threatening cerebral involvement and is the only presenting manifestation in $5 \%$ of these patients (Dupon et al., 1995). Holland et al. (1988) have reported that in $25 \%$ of AIDS patients, TRC occurs in association with encephalitis. Jabs et al. (1989) have reported that $56 \%$ of patients with ocular disease also had cerebral involvement and $12 \%$ of patients with cerebral toxoplasmosis were found to have ocular disease as well. Toxoplasma encephalitis eventually develops in $25-50 \%$ of HIV patients with antibodies against $T$. gondii (Jabs et al., 1989; Tabbara, 1990; Cochereau-Massin et al., 1992). In these individuals the disease is fatal and can even cause death, if left untreated. Toxoplasmosis was the single most common aetiological agent $(89 \%)$ of focal brain lesions at autopsy of 113 HIV patients in Bangalore, India compared to cerebral Toxoplasma lesions in 20 per cent of AIDS cases at autopsy in Mumbai, India (Shankar et al., 2005). In a clinico-radiological study of 1527 HIV seropositive subjects from Pune, western India, by Wadia et al. (2001) toxoplasmosis was the commonest cause of mass lesions $(66 \%)$.

There are several reports on application of PCR for detection of $T$. gondii in various clinical specimens like blood, cerebrospinal fluid, amniotic fluid, ocular fluids and tissue biopsy from immunocompetent and immuno suppressed patients (Dupon et al., 1995; Aouizerate et al., 1993; Garweg et al., 2000; Bou et al., 1999). The present study was to apply nested polymerase chain (nPCR) using primers targeting the B1 gene (Jones

Corresponding Author: Lily Therese, Larsen and Toubro Microbiology Research Centre, Vision Research Foundation, Chennai, Tamil Nadu, India Tel: 91-44-8271616 Fax: 91-44-8254180 
et al., 2000; Grigg et al., 2001) and SAG2 gene (Fuentes et al., 2001; Howe et al., 1997) of T. gondii on aqueous humor $(\mathrm{AH})$ and evaluate the diagnostic efficacy of these primers.

\section{MATERIALS AND METHODS}

Patients, controls and clinical specimens: The study protocol was performed in accordance with the Declaration of Helsinki. All patients and controls were recruited from Sankara Nethralaya, Chennai, India The study protocol was approved by the Ethics Committee of the institute and informed consent was obtained from each patient and control subjects. Twenty four $\mathrm{AH}$, from 12 HIV positive patients with clinically suspected Toxoplasma Retinochoroiditis (TRC) and 12 controls were included in the study. The controls included HIV positive patients with etiology proven (by PCR) acute retinal necrosis $(\mathrm{n}=6), \mathrm{CMV}$ retinitis $(\mathrm{n}=4)$ and Mycobacterium tuberculosis uveitis $(\mathrm{n}=2)$.

Processing of Aqueous Humor (AH): AH samples (150-200 $\mu \mathrm{L})$ were collected aseptically in a tuberculin syringe with a 30-gauge needle, under aseptic precautions by the ophthalmologist and $50-100 \mu \mathrm{L}$ of the sample was transferred into pre-sterilized microfuge tubes and stored at $-20^{\circ} \mathrm{C}$ for DNA extraction.

DNA extraction: DNA was extracted from standard genotype I (RH strain) strain of T. gondii and $24 \mathrm{AH}$ using DNA extraction kit (Biogene, USA) as per instructions given in the kit insert and was eluted in a final volume of $150 \mu \mathrm{L}$.

nPCR amplification: The nPCRs used in this study were standardized to detect $T$. gondii DNA. Their sensitivity was done by the standard protocol of ten fold dilution of the T. gondii DNA (RH strain), in Milli Q water and DNA concentration of the last dilution showing a positive result in nPCR is taken as the analytical sensitivity. The specificity was done using DNA extracted from bacteria $(\mathrm{n}=6)$, fungus $(\mathrm{n}=4)$, viruses $(\mathrm{n}=3)$ and parasites-Cysticercus cellulosae, Toxocara canis and Acanthameba sps.

All primers and reagents for PCR were obtained from Sanmar Speciality Chemicals Limited, (formerly Bangalore Genei Pvt. Ltd.), Bangalore, India. Two reagent controls (one for the first round amplified in the second round also and another reagent control for the second round alone) and positive control (using $2 \mu \mathrm{L}$ DNA from RH strain of $T$. gondii) were included in each PCR run. The PCR results were considered valid only when the reagent controls were negative and the specific amplified product was obtained with the positive control. To prevent contamination, DNA extraction, PCR preparation, PCR amplification and analysis of the amplified product were done in physically separated rooms. PCR preparation was performed on a laminar flow workbench with single use aliquots of reagents and dedicated pipettes. Sterile filter guarded tips were used for the addition of specimens and the extracted DNA. Visualization of PCR product was done by subjecting $10 \mu \mathrm{L}$ of amplified reaction mixture to electrophoresis on a $2 \%$ agarose gel incorporating $5 \mu \mathrm{g} \mathrm{mL} \mathrm{m}^{-1}$ of ethidium bromide in $1 \mathrm{X}$ Tris-Borate buffer ( $\mathrm{pH}$-8.2-8.6) and documented on gel documentation system (Vilber Lourmat, France).

Positive control: DNA extracted from the tachyzoites of T. gondii-RH strain, (maintained in mice by Dr. M.L. Dubey, PGIMER, Chandigarh, India) was used as positive control for $\mathrm{nPCR}$.

nPCR amplification of $B 1$ gene (primer set 1): Nested primers targeting the B1 gene (Jones et al., 2000) included outer sense primers for the first round of amplification consisting of upstream primer 5' GGA ACT GCA TCC GTT CAT GAG 3' and down stream primer 5' TCT TTA AAG CGT TCG TGG TC 3' which generates a $193 \mathrm{bp}$ product (nucleotides 694887). The inner set of II round of primers consisting of upstream primer 5' TGC ATA GGT TGC AGT CAC TG 3' and downstream primer 5' GGC GAC CAA TCT GCG AAT ACA CC 3' generate a 96 bp product (nucleotides 757-853) after amplification by nPCR. The nPCR reaction and the protocol were as given elsewhere (Mahalakshmi et al., 2006).

nPCR amplification of B1 gene (primer set 2): Nested primers coding for the B1 gene (Grigg et al., 2001) included outer sense primers for the first round of amplification consisting of upstream primer- 5'-TGT TCT GTC CTA TCG CAA CG-3' (position 127-147) and downstream primer- 5'-ACG GAT GCA GTT CCT TTC TG-3' (position 707-688) and inner set primers consists of upstream primer-5'-TCT TCC CAG ACG TGG ATT TC-3' (position 152-171) and downstream primer-5'-CTC GAC AAT ACG CTG CTT GA3 '(position 682-663). nPCR was optimized in a $50 \mu \mathrm{L}$ reaction by the method of Grigg et al. (2001) with 10 $\mu \mathrm{L}$ of DNA template of the clinical specimens in Perkin-Elmer automatic thermal cycler (Applied Biosystems, model 2400; Cetus, Norwalk, USA). Amplified product of $579 \mathrm{bp}$ product at the end of first round of amplification and/or $530 \mathrm{bp}$ product at the end of second round of amplification were considered PCR positive. 
Am. Med. J. 1 (2): 157-163, 2010

Table 1a: Primer sequence of nPCR targeting the 3' end of SAG2 gene

\begin{tabular}{ll}
\hline \multicolumn{1}{c}{ gene } & Nucleotide sequence \\
\hline Primer & 5' GACCTCGAACAGGAACAC 3' \\
Outer primer & 5', GACCTCGAACAGGAACAC 3' \\
Inner primer & 5' GAAATGTTTCAGGTTGCTGC 3' \\
& 5' GCAAGAGCGAACTTGAACAC 3' \\
\hline
\end{tabular}

Table 1b: Primer sequence of nPCR targeting the 5' end of SAG2 gene

\begin{tabular}{ll}
\multicolumn{1}{c}{ gene } \\
\hline Primer & Nucleotide sequence \\
\hline Outer primer & 5' TCTGTTCTCCGAAGTGACTCC 3' \\
& 5' TCAAAGCGTGCATTATCGC 3' \\
Inner primer & 5', ATTCTCATGCCTCCGCTTC 3' \\
& 5' AACGTTTCACGAAGGCACAC 3' \\
\hline
\end{tabular}

nPCR amplification of SAG2 gene: Two nPCRs, one targeting the 3' end of the SAG2 gene and the other targeting the 5 ' end of SAG 2 gene were optimized as per the method of Fuentes et al. (2001) The nucleotide sequence of the outer and the inner set of primers targeting the 3 'end and the 5'end of SAG2 gene are given in Table $1 \mathrm{a}$ and $1 \mathrm{~b}$ respectively. Both the nPCRS were optimized in a $50 \mu \mathrm{L}$ reaction by the method of Fuentes et al. (2001) with $10 \mu \mathrm{L}$ of DNA template of the clinical specimens in Perkin-Elmer automatic thermal cycler (Applied Biosystems, model 2400; Cetus, Norwalk, USA). For the nPCR targeting the 3' end of SAG2 gene, 300bp product at the end of first round of amplification and/or $221 \mathrm{bp}$ product at the end of second round of amplification were considered PCR positive. For the nPCR targeting the 5' end of SAG2 gene, $340 \mathrm{bp}$ product at the end of first round of amplification and/or $241 \mathrm{bp}$ product at the end of second round of amplification were considered PCR positive.

\section{RESULTS}

All the $4 \mathrm{nPCRs}$ were specific to detect only T. gondii DNA and the sensitivity was $10 \mathrm{pg}$ of T. gondii DNA for B1 gene (primer set 1), $43 \mathrm{pg}$ for B1 gene (primer set 2) and 20ag for both 3' end and 5' end of SAG2 gene respectively.

nPCR targeting B1 gene (primer set 1) was positive in $6 \mathrm{AH}, \mathrm{B} 1$ gene (primer set 2) in 2 and both 3 'end of SAG2 and 5' end of SAG2 in 4 each. All the four nPCRs were negative in the $12 \mathrm{AH}$ from the controls. The nPCR results of the $12 \mathrm{AH}$ of the patients are given in Table 2. The sensitivity of nPCR targeting B1 gene (primer set 1) is statistically significant compared to other three nPCRs (Chi square test-Yates correction: $p$ $=0.028)$. Gel Photograph showing the results of nPCR targeting B1 gene (primer set 1 and 2), 3' end and 5' end of SAG2 gene is given in Fig. 1-4 respectively.

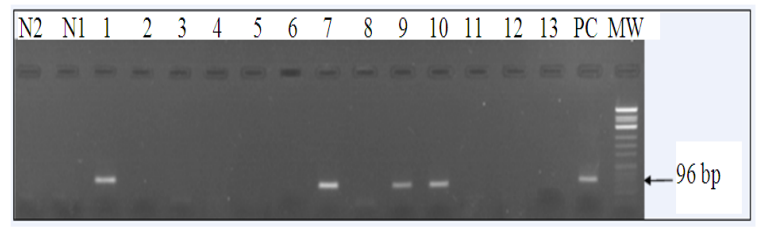

Fig. 1: Agarose gel electrophoretogram of the II round of nPCR using nested primers targeting the Blgene (primer set 1) of T. gondii genome on Aqueous Humor (AH) from 13 TRC patients. N2-Negative control II round, N1-negative control I round, (Lane 1, 7, 9 and 10) $\mathrm{AH}$ positive for T. gondii DNA; (Lane 2-6, 8, 11-13) Negative for T. gondii DNA; MW: Molecular Weight marker $\varphi X$ DNA Hinf I digest

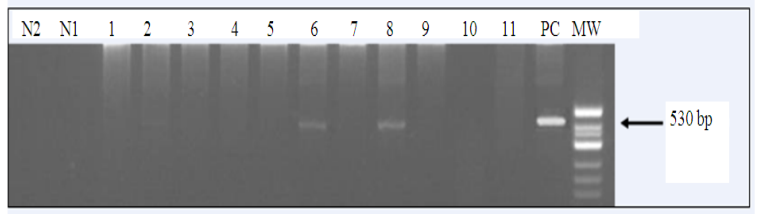

Fig. 2: Agarose gel electrophoretogram of the II round of nPCR using nested primers targeting the B1gene (primer set 2) of T. gondii genome on Aqueous Humor (AH) from 11 TRC patients. N2-Negative control II round, N1-negative control I round, (Lane 6 and 8) AH positive for T. gondii DNA; Lane 1-6, 7, 9-11-negative for T. gondii DNA; MW: Molecular Weight marker: Phi X Hinf I digest

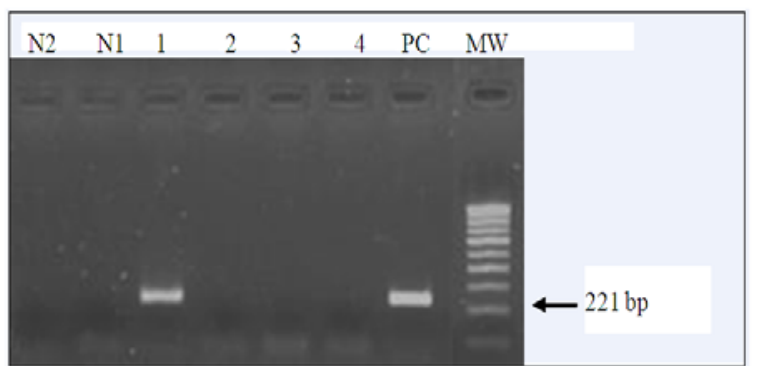

Fig. 3: Agarose gel electrophoretogram of the II round of nPCR using nested primers targeting the 3' end of SAG2 gene of $T$. gondii genome on Aqueous Humor (AH) from 4 TRC patients. N2-Negative control II round, N1-negative control I round, (Lane 1) AH positive for T. gondii DNA; (Lane 2-4) negative for T. gondii DNA; MW: Molecular Weight marker $\varphi$ X DNA Hinf I digest 
Am. Med. J. 1 (2): 157-163, 2010

Table 2: Results of nPCR targeting B1 gene (primer set 1 and 2) and SAG2 gene on AH from 12 HIV positive, clinically suspected Toxoplasma retinochoroiditis patients

\begin{tabular}{|c|c|c|c|c|c|c|c|}
\hline \multirow[b]{2}{*}{ Patient number } & \multirow[b]{2}{*}{ Age } & \multirow[b]{2}{*}{ Sex } & \multicolumn{4}{|c|}{ Results of nPCR* } & \multirow[b]{2}{*}{$\begin{array}{l}\text { Positive by one } \\
\text { or more PCR }\end{array}$} \\
\hline & & & $\begin{array}{l}\text { B1 gene } \\
\text { (primer set 1) }\end{array}$ & $\begin{array}{l}\text { B1 gene } \\
\text { (primer set 2) }\end{array}$ & $\begin{array}{l}\text { 3'- SAG2 } \\
\text { gene }\end{array}$ & $\begin{array}{l}5 \text { '- SAG2 } \\
\text { gene }\end{array}$ & \\
\hline 1 & 49 & $\mathrm{M}$ & $\mathrm{P}$ & $\mathrm{N}$ & $\mathrm{N}$ & $\mathrm{N}$ & $\mathrm{P}$ \\
\hline 2 & 38 & M & $\mathrm{P}$ & $\mathrm{N}$ & $\mathrm{N}$ & $\mathrm{N}$ & $\mathrm{P}$ \\
\hline 3 & 25 & $\mathrm{~F}$ & $\mathrm{~N}$ & $\mathrm{~N}$ & $\mathrm{~N}$ & $\mathrm{~N}$ & $\mathrm{~N}$ \\
\hline 4 & 34 & M & $\mathrm{N}$ & $\mathrm{N}$ & $\mathrm{P}$ & $\mathrm{P}$ & $\mathrm{P}$ \\
\hline 5 & 48 & M & $\mathrm{P}$ & $\mathrm{N}$ & $\mathrm{N}$ & $\mathrm{N}$ & $\mathrm{P}$ \\
\hline 6 & 34 & M & $\mathrm{N}$ & $\mathrm{N}$ & $\mathrm{N}$ & $\mathrm{N}$ & $\mathrm{N}$ \\
\hline 7 & 38 & M & $\mathrm{P}$ & $\mathrm{P}$ & $\mathrm{P}$ & $\mathrm{P}$ & $\mathrm{P}$ \\
\hline 8 & 37 & $\mathrm{M}$ & $\mathrm{N}$ & $\mathrm{N}$ & $\mathrm{P}$ & $\mathrm{P}$ & $\mathrm{P}$ \\
\hline 9 & 33 & M & $\mathrm{P}$ & $\mathrm{N}$ & $\mathrm{N}$ & $\mathrm{N}$ & $\mathrm{P}$ \\
\hline 10 & 35 & M & $\mathrm{P}$ & $\mathrm{P}$ & $\mathrm{P}$ & $\mathrm{P}$ & $P$ \\
\hline 11 & 32 & $\mathrm{M}$ & $\mathrm{N}$ & $\mathrm{N}$ & $\mathrm{N}$ & $\mathrm{N}$ & $\mathrm{N}$ \\
\hline 12 & 45 & $\mathrm{M}$ & $\mathrm{N}$ & $\mathrm{N}$ & $\mathrm{N}$ & $\mathrm{N}$ & $\mathrm{N}$ \\
\hline No. of samples $p$ & & & 6 & 2 & 4 & 4 & 8 \\
\hline
\end{tabular}

*: The sensitivity of nPCR targeting B1 gene (primer set 1) is statistically significant compared to other nPCR (Chi square test-Yates correction: $\mathrm{p}=0.028$

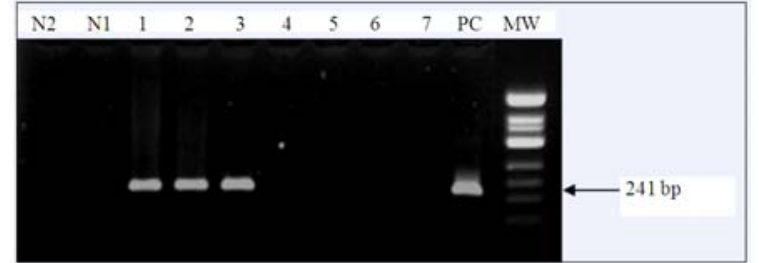

Fig. 4: Agarose gel electrophoretogram of the II round of nPCR using nested primers targeting the Blgene (primer set 1) of T. gondii genome on Aqueous Humor (AH) from 13 TRC patients. N2-Negative control II round, N1-negative control I round, (Lane 1, 7, 9 and10) $\mathrm{AH}$ positive for T. gondii DNA; (Lane 2-6, 8, 11-13) negative for T. gondii DNA; MW: Molecular Weight marker: 100 bp ladder

\section{DISCUSSION}

Currently the clinical diagnosis of ocular toxoplasmosis is based on the observation of a typical necrotizing lesion on the fundus, response to treatment and serological diagnosis (Jones et al., 2000; Garweg et al., 1996). In HIV positive patients, differentiation of Toxoplasma retinochoroiditis, from other disorders is most difficult, as lesions (i) can present with a variety of clinical features, (ii) are not usually associated with retinal scars and (iii) may result in wide spread areas of retinal necrosis that resemble other infections, most commonly CMV retinitis and acute retinal necrosis syndrome (Moorthy et al., 1993; Estrid et al., 2000; Miller et al., 2000). In such cases the aqueous humor analysis is being used as a diagnostic tool for confirmation of TRC (Bou, et al., 1999; Miller et al., 2000). Molecular technique-Polymerase Chain Reaction (PCR) for the detection of T. gondii genome in ocular fluid blood is a rapid sensitive and reliable diagnostic technique.

B1 gene is a 35 fold repetitive conserved gene within the T. gondii genome and was detected among all the strains of $T$. gondii tested till date including several isolates from AIDS patients (Jones et al., 2000; Miller et al., 2000; Brezin et al., 1990). B1 gene was identified by Boothroyd and associates, contains an intron and does not encode a known protein (Brezin et al., 1990) and nPCR targeting B1 gene has been proved to be both specific and sensitive of the primers tested, (Jones et al., 2000; Miller et al., 2000; Brezin et al., 1990; Robert-Gangneux et al., 1999) making it ideal target for PCR amplification (Chan et al., 1994; Manners et al., 1994). The analytical sensitivity of one tachyzoite by nPCR targeting B1 gene in this study is similar to that of other authors (Jones et al., 2000; Moorthy et al., 1993). Surface antigen 2 (SAG2) gene codes for the protein P22 of $T$. gondii, which is a major surface protein known as an attachment ligand that also has good antigenicity and immunogenicity (Fuentes et al., 2001) SAG2 gene is ideally suited for rapid genotyping, as it contains multiple lineage-specific polymorphisms. SAG2 encodes two separate forms of the surface tachyzoite protein p22 that are recognized by strainspecific monoclonal antibodies: type I and III strains share the same protein allele, while type II strains have a second, distinct form (Howe et al., 1997). The usefulness of nPCR targeting SAG2 gene directly on clinical specimens has already been studied (Fuentes et al., 2001; Howe et al., 1997).

All the $4 \mathrm{nPCRs}$ in this study were done on the same DNA and the PCRs were also done almost at the same time, which also rules out degradation of DNA due to storage. The advantage of in vitro amplification systems is their high analytical sensitivity. However, 
high analytical sensitivity does not necessarily translate into high clinical sensitivity (Persing, 1994). In this study, the 2 nPCRs targeting B1 gene had different analytical and clinical sensitivity. The sensitivity of nPCRs targeting SAG2 gene was higher compared to the B1 gene (Primer set 1), but the clinical sensitivity of B1 gene (primer set 1) was higher than SAG2 gene. The volume of sample tested has a direct bearing on clinical performance if the target copy number is low. The effects of the inhibitors are themselves amplified in a sample containing low copy numbers of the target sequence. Thus, it is important to differentiate analytical sensitivity and clinical sensitivity in the assessment of an amplification technology for clinical use and to realize that an amplification system may not always provide adequate performance despite having high analytical sensitivity (Persing, 1994).

Only the ability to detect $T$. gondii DNA in ocular samples provide direct evidence of the infection as neither culture not the serological tests are useful in immuno compromised individual (Jones et al., 2000). Immuno-deficient patients have markedly higher parasite DNA copy numbers and manifest a feebler immunological degradation of target DNA than immunocompetent patients. Furthermore, the ocular disease in immuno-deficient individuals is an acute form of generalized infection, which is not immunologically controlled. Consequently, the probability of detecting parasite or their DNA in any bodily compartment of immuno deficient patient is much higher than that for immuno competent individuals with a previous or chronic infection (Garweg et al., 2000). In this study, T. gondii DNA was detected by one or more of the $4 \mathrm{nPCRs}$ in $66.7 \%$ of the 12 patients.

In a study by De-Boer et al. (1996), on 7 immunosuppressed patients with TRC, PCR targeting rDNA, was positive in $42.9 \%$ (3/7) patients compared to a sensitivity of $50 \%$ of $\mathrm{B} 1$ gene (primer set 1 ), $33.3 \%$ of SAG2 gene $16 \%$ of B1 gene (primer set 2) in this study. Inter-laboratory variability is a confounding factor when analyzing results and trying to compare different methodologies used at different centers (Jones et al., 2000). Predominant of the reports of toxoplasmosis in HIV patients in India, (Shankar et al., 2005; Wadia et al., 2001) Brazil (Colombo et al., 2005; Vidal et al., 2004) and USA(Khan et al., 2005) are on cerebral toxoplasmosis, which is more commoner and life threatening in HIV positive patients. In India, Toxoplasma is the most common agent causing focal mass lesions in brain among HIV positive patients (Shankar et al., 2005). A prospective study on a larger scale will throw more light on the prevalence of TRC and Toxoplasma encephalitis and the genotype of T. gondii among the HIV patients in India.

\section{CONCLUSION}

Among the 4 nPCRs, nPCR targeting $\mathrm{B} 1$ gene (primer set 1) was the most sensitive, and reliable nucleic acid amplification technique for the laboratory diagnosis of TRC in HIV patients.

\section{ACKNOWLEDGEMENT}

The authors gratefully acknowledge the financial support of Indian Council of Medical Research, through the grant (Project no: 5/3/3/3/ 2003 ECD-I). We acknowledge Dr. M.L. Dubey, PGIMER, Chandigarh, India for the supply of inactivated standard RH strain of T. gondii.

\section{REFERENCES}

Aouizerate, F., J. Cazenave, L. Poirier, P.H. Verin and A. Cheyrou et al., 1993. Detection of Toxoplasma gondii in aqueous humor by the polymerase chain reaction. Br. J. Ophthalmol., 77: 107-109. DOI: 10.1136/bjo.77.2.107

Bou, G., M.S. Firueroa, P.M. Belda, E. Navas and A. Guerrero, 1999. Value of PCR for detection of Toxoplasma gondii in aqueous humor and blood samples from immunocompetent patients with ocular toxoplasmosis. J. Clin. Microbiol., 37: 3465-3468. PMID: 10523535

Brezin, A.P., C.E. Egwuagu, M. Burnier, C. Silveira and R. Mahdi et al., 1990. Identification of Toxoplasma gondii in paraffin-embedded sections by polymerase chain reaction. Am. J. Ophthalmol., 110: 599-604. PMID: 2248322

Chan, C.C., A.G. Palestine, Q. Li and R.B. Nussenblatt, 1994. Diagnosis of ocular toxoplasmosis by the use of immunocytology and the polymerase chain reaction. Am. J. Ophthalmol., 117: 803-805. PMID: 7911007

Cochereau-Massin, I., P. LeHoang, M. Lautier-Frau, E. Zerdoun and L. Zazoun et al., 1992. Ocular toxoplasmosis in human immunodeficiency virusinfected patients. Am. J. Ophthalmol., 114: 130-135. PMID: 1322640

Colombo, F.A., J.E. Vidal, A.C.P. de-Oliveira, A.V. Hernandez and F. Bonasser-Filho et al., 2005. Diagnosis of cerebral toxoplasmosis in AIDS patients in Brazil: Importance of molecular and immunological methods using peripheral blood samples. J. Clin. Microbiol., 43: 5044-5047. DOI: 10.1128/JCM.43.10.5044-5047.2005 
De-Boer, J.H., C. Verhagen, M. Bruinenberg, A. Rothova and P.T.V.M. De-Jong et al., 1996. Serologic and polymerase chain reaction analysis of intraocular fluids in the diagnosis of infectious uveitis. Am. J. Ophthalmol., 121: 650-658. PMID: 8644808

Dupon, M., J. Cazenave, J.L. Pellegrin, J.M. Ragnaud and A. Cheyrou et al., 1995. Detection of Toxoplasma gondii by PCR and tissue culture in cerebrospinal fluid and blood of human immunodeficiency virus-seropositive patients. J. Clin. Microbiol., 33: 2421-2426. PMID: 7494040

Estrid, H., V. Jens, L. Peter and P. Eskild, 2000. Characterization of Toxoplasma gondii isolates using Polymerase Chain Reaction (PCR) and Restriction Fragment Length Polymorphism (RFLP) of the Toxoplasma Gondii (TGR) gene sequences. Int. J. Parasitol., 30: 853-858. DOI: 10.1016/S0020-7519(00)00059-X

Fuentes, I., J.M. Rubio, C. Ramirez and J. Alvar, 2001. Genotypic characterization of Toxoplasma gondii strains associated with human toxoplasmosis in Spain: Direct analysis from clinical specimens. J. Clin. Microbiol., 39: 1566-1570. DOI: 10.1128/JCM.39.4.1566-1570.2001

Garweg, J., M. Boehnke and F. Koerner, 1996. Restricted applicability of the polymerase chain reaction for the diagnosis of ocular toxoplasmosis. German. J. Ophthalmol., 5: 104-108. PMID: 8741155

Garweg, J.G., P. Jacquer and M. Boehnke, 2000. Early aqueous humor analysis in patients with human ocular toxoplasmosis. J. Clin. Microbiol., 38: 996-1001. PMID: 10698986

Grigg, M.E., S. Bonnefoy, A.B. Hehl, Y. Suzuki and J.C. Boothroyd, 2001. Success and virulence in Toxoplasma as the result of sexual recombination between two distinct ancestries. Science, 294: 161-165. DOI: $10.1126 /$ science. 1061888

Holland, G.N., R.E. Engstrom and B.J. Glasgow, 1988. Ocular toxoplasmosis in patients with the acquired immuno deficiency syndrome. Am. J. Ophthalmol., 106: 653-667. PMID: 3195645

Holland, G.N., 1999. Reconsidering the pathogenesis of ocular toxoplasmosis. Am. J. Ophthalmol., 128: 502-505. DOI: $10.1016 / \mathrm{S} 0002-$ 9394(99)00263-9

Howe, D.K., S. Honore, F. Deouin and L.D. Sibley, 1997. Determination of genotypes of Toxoplasma gondii strains isolated from patients with toxoplasmosis. J. Clin. Microbiol, 35: 1411-1414. PMID: 9163454

Jabs, D.A., W.R. Green and R. Fox, 1989. Ocular manifestations of acquired immune deficiency syndrome. Ophthalmology, 96: 1092-1099. DOI: 10.1097/00004397-198502520-00019
Jones, C.D., N. Okhravi, P. Adamson, S. Tasker and S. Lightman, 2000. Comparison of PCR detection methods for B1, P30 and 18S rDNA genes of Toxoplasma gondii in aqueous humor. Invest. Ophthalmol. Vis. Sci., 41: 634-644. PMID: 10711675

Khan, A., C. Su, M. Geraman, G.A. Storch, D. Clifford and L.D. Sibley, 2005. Genotyping of Toxoplasma gondii strains from immunecompromised patients reveal high prevalence of type I strains. J. Clin. Microbiol., 43: 5881-5887. DOI: $10.1128 /$ JCM.43.12.5881-5887.2005

Mahalakshmi, B., K.L. Therese, H.N. Madhavan and J. Biswas, 2006. Diagnostic value of specific local antibody production and nucleic acid amplification technique-nested Polymerase Chain Reaction (nPCR) in clinically suspected ocular toxoplasmosis. Ocul. Immunol. Inflam., 14: 105-112. DOI: 10.1080/09273940500545692

Manners, R.M., S.O. Connell, E.C. Guy, D.H.M. Joynson, C.R. Canning et al., 1994. Use of polymerase chain reaction in the diagnosis of acquired ocular toxoplasmosis in an immunocompetent adult. Br. J. Ophthalmol., 78: 583-584. DOI: 10.1136/bjo.78.7.583

Miller, D., J. Davis, R. Rosa and E. Perez, 2000. Utility of tissue culture for detection of Toxoplasma gondii in vitreous humor of patients diagnosed with Toxoplasma retinochoroiditis. J. Clin. Microbiol., 38: 3840-3842. PMID: 11015415

Moorthy, R.S., R.E. Smith and N.A. Rao, 1993. Progressive ocular toxoplasmosis in patients with acquired immunodeficiency syndrome. Am. J. Ophthalmol., 115: 742-747. PMID: 8506909

Persing, D.H., 1994. In Vitro Nucleic Acid Amplification Technique. In: Diagnostic Molecular Microbiology: Principles and Application, Persing, D.H., T.F. Smith, F.C. Tenover and T.J. White (Eds.). American Society for Microbiology, Washington DC., pp: 51-88. ISBN: 155581221X; ISBN-13: 9781555812218

Robert-Gangneux, F., M.F. Gavinet, T. Ancelle, J. Raymond, C.T. Shaefer et al., 1999. Value of prenatal diagnosis and early postnatal diagnosis of congenital toxoplasmosis: Retrospective study of 110 patients. J. Clin. Microbiol., 37: 2893-2898. PMID: 10449471

Shankar, S.K., A. Mahadevan, P. Satishchandra, R.U. Kumar and T.C. Yasha et al., 2005. Neuropathology of HIV/AIDS with an overview of the Indian scene. Indian. J. Med. Res., 121: 468-488. PMID: 15817957

Tabbara, K.F., 1990. Ocular toxoplasmosis. Int. Ophthalmol., 14: 349-351. DOI: 10.1007/BF00163555 
Vidal, J.E., F.A. Colombo, A.C.P. de-Oliveira, R. Focaccia et al., 2004. PCR assay using cerebrospinal fluid for diagnosis of cerebral toxoplasmosis in Brazilian AIDS patients. J. Clin. Microbiol., 42: 4765-4768. DOI: 10.1128/JCM.42.10.4765-4768.2004
Wadia, R.S., S.N. Pujari, S. Kothari, M. Udhar and S. Kulkarni et al., 2001. Neurological manifestations of HIV disease. J. Assoc. Phys. India, 49: 343-348. PMID: 11291974 